\title{
Acute-on-chronic liver failure: a review
}

This article was published in the following Dove Press journal:

Therapeutics and Clinical Risk Management

23 April 2014

Number of times this article has been viewed

Luis Eduardo Zamora Nava' Jonathan Aguirre Valadez ${ }^{2}$ Norberto C Chávez-Tapia ${ }^{3}$ Aldo Torre ${ }^{2}$

'Department of Endoscopy, ${ }^{2}$ Department of Gastroenterology, National Institute of Medical Sciences and Nutrition Salvador Zubirán, ${ }^{3}$ Obesity and Digestive Diseases Unit, Medica Sur Clinic and Foundation, Mexico City, Mexico
Correspondence: Aldo Torre Vasco de Quiroga 15, Colonia Sección XVI, Tlalpan CPI4000, Mexico DF, Mexico Tel +52 5554870900 Email detoal@yahoo.com
Abstract: There is no universally accepted definition of acute-on-chronic liver failure; however, it is recognized as an entity characterized by decompensation from an underlying chronic liver disease associated with organ failure that conveys high short-term mortality, with alcoholism and infection being the most frequent precipitating events. The pathophysiology involves inflammatory processes associated with a trigger factor in susceptible individuals (related to altered immunity in the cirrhotic population). This review addresses the different definitions developed by leading research groups, epidemiological and pathophysiological aspects, and the latest treatments for this entity.

Keywords: acute-on-chronic liver failure, cirrhosis, organ failure, acute kidney injury, infection

\section{Introduction}

Cirrhosis represents the end stage of chronic liver disease that is accompanied by progressive fibrosis and is characterized by development of regenerative nodules and a sustained chronic inflammatory response. ${ }^{1}$ Once established, cirrhosis is irreversible, and is associated with increased morbidity, development of life-threatening complications, and decreased quality of life.

The clinical course of the disease is characterized by a "compensated" or asymptomatic phase followed by a rapidly progressive phase "decompensated", resulting in development of complications (jaundice, variceal hemorrhage, ascites, encephalopathy) secondary to the two main syndromes associated with cirrhosis, ie, portal hypertension and hepatic insufficiency. ${ }^{2,3}$ Therefore, cirrhosis is classified into four stages, ie, two compensated stages (absence of ascites or presence of nonhemorrhagic esophageal varices) and two decompensated stages (presence of ascites and variceal bleeding), which are related to hemodynamic changes in terms of portal pressure gradient and histological stages of fibrosis. ${ }^{2,4}$ Recently, a fifth stage has been proposed, that consists of infection (sepsis) in critically ill patients, and its presence has been associated with increased mortality ${ }^{5}$ (Figure 1).

Cirrhotic patients may have stable liver function for long periods of time, and an acute insult in the presence of advanced fibrosis and decreased functional reserve may lead to development of hepatic decompensation. Clinical practice shows that these patients may develop decompensation in two ways. The first and most common one is a progressive decompensation resulting in a clinical course of end-stage liver disease (chronic liver decompensation), and the second is acute liver decompensation resulting from a precipitating event (acute insult), such as variceal bleeding, alcoholism, or sepsis 


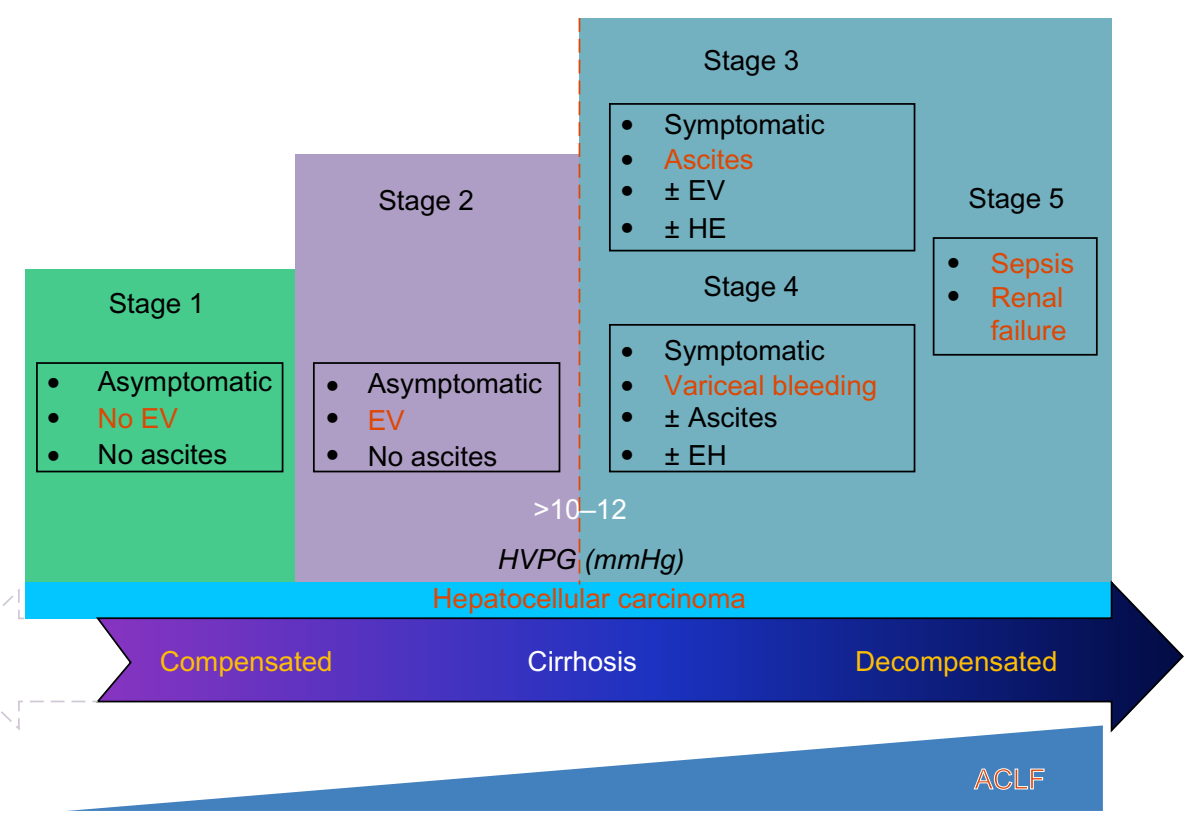

Figure I Clinical course of cirrhosis.

Abbreviations: EV, esophageal varices; HE, hepatic encephalopathy; HVPG, hepatic venous pressure gradient; ACLF, acute-on-chronic liver failure.

in patients with previously compensated liver disease. Here, we will use the term acute-on-chronic liver failure (ACLF) for an entity characterized by rapid progression requiring support for multiple organ failure and a high mortality rate $(50 \%-90 \%)$ in the short or medium term. ${ }^{6-8}$ It is very important to distinguish between chronic decompensation resulting from progression of chronic liver disease, which in most cases is irreversible, and ACLF, which may be reversible if the trigger factor is treated. ${ }^{6-10}$ It is noteworthy that liver disease and a precipitating event are found in both ACLF and acute liver decompensation, and organ failure associated with high short-term mortality in ACLF is the only difference between these two conditions. ${ }^{11}$

There is a growing need to study this "new" clinical entity, mainly because of an increase in the number of patients with chronic liver disease who are at risk of developing an episode of acute liver decompensation during their clinical course.

\section{Definition}

The term ACLF was first introduced in 1995 to describe a condition in which two "lesions" or "insults" act simultaneously on the liver, one of which is chronic and the other is acute. ${ }^{12,13}$ However, there is no clear definition, and previous studies have used this term to refer to any chronic liver disease (compensated or not) associated with an acute event but without specifying the spectrum of chronic liver disease.

Therefore, several definitions have been developed by expert groups from the American Association for the Study of Liver Diseases (AASLD),${ }^{14}$ European Association for the Study of the Liver (EASL), ${ }^{14}$ and Asian-Pacific Association for the Study of the Liver (APASL). ${ }^{13}$

The definition proposed by the AASLD and EASL working group is: "acute deterioration of preexisting chronic liver disease usually related to a precipitating event and associated with increased mortality at 3 months due to multi-system organ failure". ${ }^{14}$ On the other hand, the group of experts from APASL propose the following operational definition: "acute liver injury manifesting as jaundice (serum bilirubin $\geq 5 \mathrm{mg} / \mathrm{dL}$ ) and coagulopathy (INR $\geq 1.5$ or prothrombin activity of $<40 \%$ ), complicated within 4 weeks by ascites and/or encephalopathy in a patient previously diagnosed or undiagnosed chronic liver disease". ${ }^{13}$

Despite the proposed ACLF definitions and given the lack of consensus, a group of researchers from EASL-Chronic Liver Failure (CLIF) Consortium recently carried out a prospective multicenter study to evaluate the characteristics of individuals with liver disease and acute decompensation to determine factors associated with mortality, and found that patients with acute liver decompensation who had organ failure and high mortality rates in the short term (28 days) could be diagnosed with ACLF. ${ }^{11}$ Interestingly, ACLF may occur even in the absence of liver failure (defined as bilirubin level $\geq 12 \mathrm{mg} / \mathrm{dL}$ unlike the $5 \mathrm{mg} / \mathrm{dL}$ proposed by APASL). ${ }^{11,13}$ This study also proposed three groups of ACLF according to mortality rate, which depends on the organ involved in organ failure and the number of such failures. ${ }^{11}$ 


\section{Epidemiology}

There are few epidemiological studies regarding this entity. According to results presented at the EASL-AASLD Single Topic Symposium, 26,300 cirrhotic patients required mechanical ventilation and/or invasive cardiovascular monitoring, with an inhospital mortality rate of $53 \%$ and an average inhospital stay of 14 days, ${ }^{14}$ and it is likely that a significant number of those patients had ACLF. In a recent study conducted in Europe involving 1,343 patients hospitalized for acute liver decompensation with an underlying diagnosis of cirrhosis, the prevalence of ACLF was $30.9 \%$ and was associated with a short-term (28-day) mortality 15 times higher than in cirrhotic patients without ACLF. ${ }^{11}$

\section{Chronic liver disease and acute liver injury}

The first element involved in the definition of ACLF is the presence of chronic liver disease, which is defined by the APASL as the presence of compensated cirrhosis of any etiology including, in decreasing order, cholestatic and metabolic liver disease, chronic hepatitis, and nonalcoholic steatohepatitis, and excluding liver steatosis. ${ }^{13}$

Another key element in the definition of ACLF is the presence of a precipitating event, ie, a new acute insult, related or not to the liver disease, which will be addressed in more detail later. ${ }^{14}$ In a study conducted by Duseja et al that included 102 patients with ACLF, the most common precipitating factor observed was infection. ${ }^{15}$

\section{Pathophysiology}

The transition from compensated cirrhosis to ACLF is accompanied by a systemic inflammatory response characterized by a predominantly proinflammatory cytokine profile, which is believed to mediate inflammation, apoptosis and necrosis of liver cells, as well as fibrosis and cholestasis. ${ }^{10,13,16,17}$ The pathophysiology of ACLF can be approached using the PIRO (predisposition, infection, response and organ failure) system that is commonly used to describe the pathophysiology of sepsis. ${ }^{18}$

\section{Precipitating event}

Precipitating events vary according to geographic region and study population. For example, alcohol and drugs are predominant factors in Western countries, whereas infectious etiologies (hepatitis B) are dominant factors in Eastern countries. ${ }^{13}$ Precipitating events that may affect and worsen liver function can be divided into two groups: the first group involves events that directly affect liver function, ie, drug-induced liver injury, alcoholic hepatitis, new-onset viral hepatitis, and ischemic hepatitis (mainly Budd Chiari syndrome); the second group involves systemic events (extrahepatic) that have repercussions for liver function (surgery, infection, trauma, or variceal bleeding). ${ }^{19}$

In the study conducted by Duseja et al, the most common precipitating factor observed was infection, seen in up to $53 \%$ of cases; the most common infections were spontaneous bacterial peritonitis and sepsis in $47 \%$ of cases and viral hepatitis in the remaining $6 \% .{ }^{15}$ In a recent study by EASL-CLIF, ${ }^{11}$ the precipitating event could not be identified in $43 \%$ of patients with ACLF; interestingly, even in cases in which the precipitating event was determined, the event was not associated with severity of ACLF or short-term mortality.

In patients undergoing surgery, the most important predictors of postoperative mortality were severity of liver disease as determined by MELD (Model for End-stage Liver Disease) score, age, and the score given by the American Society of Anesthesiologists. ${ }^{20}$

Recently, the RELIEF trial conducted in the European community which evaluated use of extracorporeal albumin dialysis with the molecular adsorbent recirculating system in ACLF found that the most common precipitating factors were alcohol abuse and infection, and up to $38 \%$ of patients had more than one precipitating factor. ${ }^{21}$

\section{Inflammation: response and infection}

In ACLF, hepatocytes are exposed to elevated levels of inflammatory cytokines, ie, interleukin (IL)-2, IL-6, interferon- $\gamma$, and tumor necrosis factor- $\alpha$, which simultaneously activate survival and apoptosis pathways depending on levels of proapoptotic or antiapoptotic activity. ${ }^{22}$

Both apoptosis and necrosis represent two main patterns of cell death. While apoptosis is the main mechanism involved in acute and chronic liver damage, ${ }^{23}$ the term "necroapoptosis" has been introduced recently, indicating that these two forms of cell death have common pathways and are not mutually exclusive entities, where the pattern of death cell can change from one extreme to another in the same individual, supporting the idea of programmed and controllable necrosis. ${ }^{24}$

The similarity between ACLF and severe sepsis/septic shock has led to the concept of "immune paralysis" as the mechanism underlying both conditions. Immune paralysis consists of decreased expression of human leukocyte antigen in monocytes, thus altering the lipopolysaccharide-mediated production of proinflammatory cytokines (IL-1, IL-6, IL-8, tumor necrosis factor- $\alpha)^{25}$ 
Another important aspect observed in patients with ACLF (of alcoholic etiology) is alterations in neutrophil function secondary to endotoxemia, such as increased basal oxidative tone and decreased phagocytic capacity. ${ }^{26}$ Interestingly, preliminary results suggest that modulation of gut flora favors the recovery of phagocytic capacity. ${ }^{27}$ All this could contribute to an increased risk of infection, worsening of progressive vasodilation status, development of multiple organ failure, and increased mortality in this group of patients. ${ }^{13,28,29}$

In cirrhotic patients, the presence of sepsis is a common precipitating event, resulting in liver decompensation, kidney dysfunction, and mortality associated with variceal bleeding. About $40 \%-50 \%$ of patients with cirrhosis admitted to hospital have sepsis, and 20\%-40\% of patients will develop nosocomial infections, with an observed mortality of $15 \%$, which is twice the proportion of mortality compared with patients who have cirrhosis without infection. 5,30

Rastogi et a ${ }^{31}$ recently found an increased number of activated hepatic stellate cells in patients with ACLF compared with patients who had acute hepatitis and chronic liver disease. Moreover, they showed a significant relationship between numbers of hepatic stellate cells and liver stem cells, indicating a potential dynamic role of hepatic stellate cells in liver regeneration and possibly in the pathophysiology of ACLF.

\section{Organ failure}

According to the results of the current studies, it appears that the degree and number of organ failures are the major factors determining the outcome (mortality) of patients with ACLF, and not the degree of liver disease, as determined by conventional (Child Pugh) scores. ${ }^{11}$ Organ failure is a major factor in the clinical course of ACLF. In fact, organ failure and number of organ failures enabled identification of three groups of ACLF in the EASL-CLIF study, each characterized by higher mortality (Table 1).

\section{Liver damage}

Two distinctive features of ACLF are hyperbilirubinemia and coagulopathy, although the pathophysiological basis is still unclear. Elevated bilirubin levels have been directly associated with increased risk of infection, which has been a major cause of mortality in this group. ${ }^{32}$ Some studies have demonstrated the role of endotoxemia and bacterial translocation in the pathogenesis of portal hypertension. Modification of the intestinal bacterial load by use of anti-
Table I

\begin{tabular}{|c|c|}
\hline \multirow[t]{2}{*}{ ACLF grade } & Characteristics \\
\hline & Acute liver damage associated with \\
\hline \multirow[t]{5}{*}{ Grade I } & Single kidney failure ${ }^{a}$ OR liver failure, ${ }^{b}$ \\
\hline & coagulopathy, ${ }^{\mathrm{c}}$ circulatory failure, ${ }^{\mathrm{d}}$ respiratory \\
\hline & failure, ${ }^{e}$ serum creatinine $1.5-1.9 \mathrm{mg} / \mathrm{dL}$ and/or \\
\hline & mild to moderate hepatic encephalopathy OR \\
\hline & brain failure ${ }^{f}$ with creatinine $1.5-1.9 \mathrm{mg} / \mathrm{dL}$ \\
\hline Grade 2 & Two organ failures \\
\hline Grade 3 & Three or more organ failures \\
\hline
\end{tabular}

Notes: CLIF-SOFA criteria (modified SOFA score considering aspects of chronic liver damage; this score was validated to be used in this study, based on authors' experience) per organ; ${ }^{a}$ serum creatinine $\geq 2 \mathrm{mg} / \mathrm{dL}$ or kidney replacement therapy; ${ }^{b}$ total bilirubin $\geq 12$; International Normalized Ratio $\geq 2.5$ or platelets $20 \times 10^{9} / \mathrm{L}$; use of dopamine, dobutamine, terlipressin, norepinephrine, epinephrine; e $\mathrm{PaO} / \mathrm{FiO}_{2} \leq 200 \mathrm{mg} / \mathrm{dL}$ or $\mathrm{SpO}_{2} / \mathrm{FiO}_{2} \leq 214 \mathrm{mg} / \mathrm{dL}$; 'hepatic encephalopathy grade III or IV. Data from from Moreau et al."

Abbreviations: ACLF, acute-on-chronic liver failure; CLIF, Chronic Liver Failure; SOFA, Sequential Organ Failure Assessment.

biotics, such as quinolones and rifaximin, has been associated with a decrease in portal pressure and the degree of inflammation. ${ }^{33}$

In a prospective study by Moreau et al, ${ }^{11}$ it was observed that the presence of liver failure (as defined by CLIF-SOFA [Sequential Organ Failure Assessment] score) carries a low risk of death unless it is associated with kidney dysfunction or hepatic encephalopathy (moderate to severe). On the other hand, in a study by Bañares et al, it was found that prognostic factors in patients with ACLF were degree of liver damage as determined by MELD score, a greater degree of encephalopathy at baseline, and increased bilirubin during the first 4 days, ${ }^{21}$ showing the criteria for defining the impact of ACLF on the reported results and outcomes.

\section{Kidney damage}

Kidney dysfunction in the presence of ACLF may be different to that in the presence of hepatorenal syndrome. Circulatory changes may be found in some patients, whereas an increase in synthesis of proinflammatory mediators may be observed in others, and in some cases both situations are present. Acute kidney injury is an alteration frequently associated with ACLF and carries a poor prognosis. The role of inflammation as a modulator of this entity is manifested by the reduced risk of renal dysfunction in patients with alcoholic hepatitis using anti-inflammatory agents such as albumin, pentoxifylline, and N-acetylcysteine. ${ }^{34,35}$ In the EASL-CLIF Consortium study, it was found that type of organ failure was clearly associated as a mortality risk factor, and was higher in the subgroup of patients with single kidney failure than in those with involvement of other organs. ${ }^{11}$ 


\section{Other organs}

With regard to brain damage, hepatic and hyponatremic encephalopathies are the most frequently observed conditions, and in some cases can coexist. ${ }^{36}$ In an intensive care unit, a mortality rate of $10 \%$ was reported in patients with liver encephalopathy alone and a mortality of $80 \%$ in those with involvement of other organs. ${ }^{37}$ In the study by Bañares et al, it was found that the highest degree of encephalopathy was one of the prognostic factors in patients with ACLF. ${ }^{21}$ Cardiovascular collapse is associated with increased mortality in patients with ACLF, mainly in association with kidney failure. $^{38}$

\section{Prognosis and clinical course}

The prognosis and clinical course vary from one patient to another, and depend not only on the magnitude of the acute injury, but also the degree of functional hepatic reserve (hypothesis of multiple lesions and critical mass). ${ }^{10,13,14}$ Like acute liver failure, ACLF is a condition with multisystemic involvement where the main trigger factor is a systemic inflammatory response caused most often by an infectious process triggering mainly hemodynamic (hyperdynamic circulation, portal hypertension), ${ }^{39}$ neurological (liver encephalopathy), ${ }^{40,41}$ and kidney (acute kidney injury/ hepatorenal syndrome) disturbances ${ }^{42}$ (Figure 2). Patients with ACLF have a short-term mortality of $50 \%-90 \%,{ }^{43-45}$ and when compared with that observed in cirrhotic patients without ACLF, even with the same MELD scoring, mortality is higher in ACLF. ${ }^{14}$

Regarding the prognostic scales used to assess decompensated cirrhotic patients admitted to the intensive care unit, there are two main types of models, ie, those that assess the severity of disease (Acute Physiology and Chronic Health Evaluation [APACHE], Simplified Acute Physiology Score, and Mortality

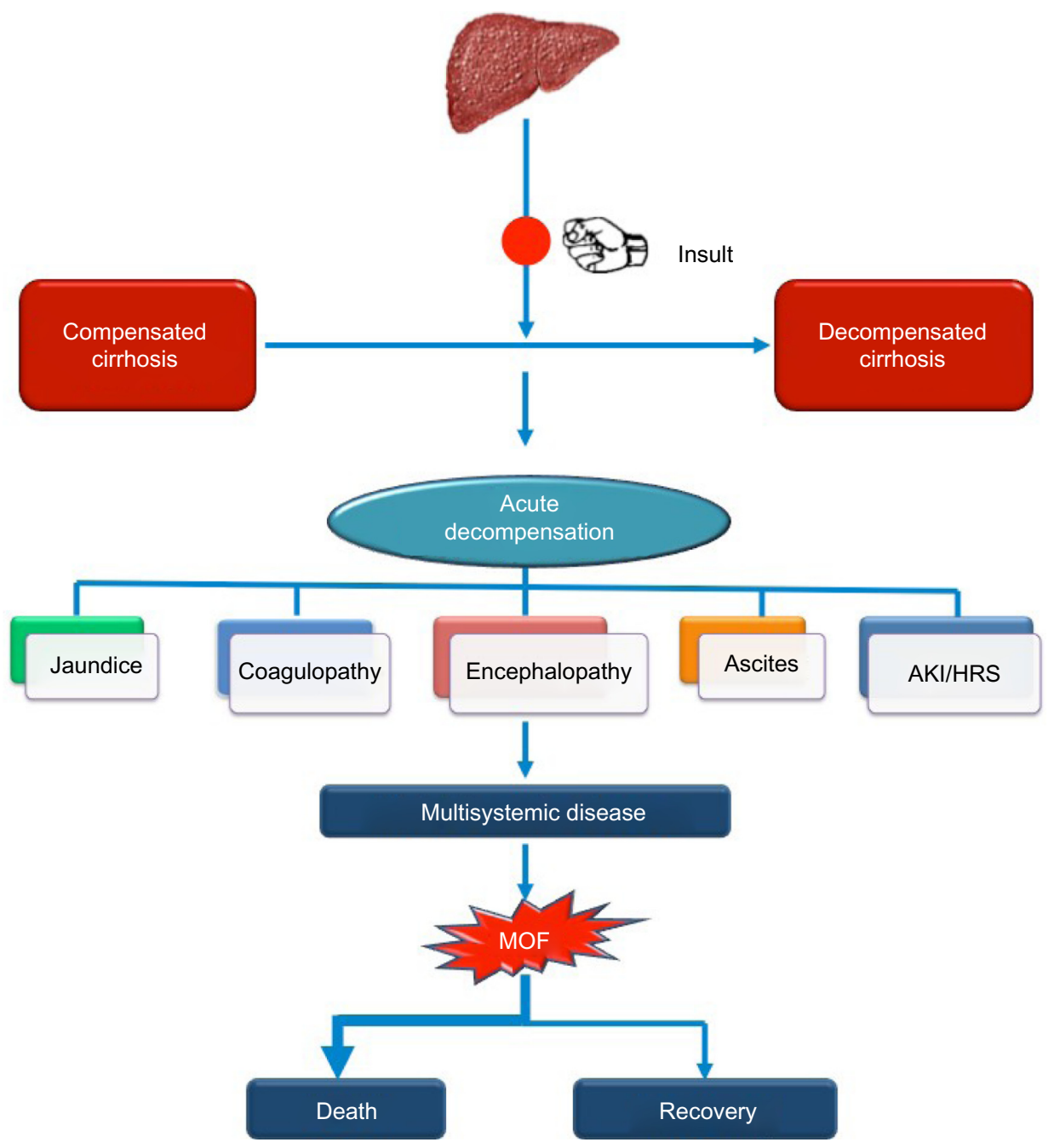

Figure 2 Clinical course of acute-on-chronic liver failure.

Abbreviations: AKI, acute kidney injury; HRS, hepatorenal syndrome; MOF, multiple organ failure. 
Prediction Model) and those that quantify the degree of organ failure (Logistic Organ Dysfunction System, Multiple Organ Dysfunction Score, Organ System Failure, and SOFA), which in general have greater predictive ability than the Child-Pugh score. ${ }^{46}$ A study involving 184 patients with ACLF admitted to the intensive care unit found that baseline APACHE II and MELD scores, and development of bacteremia were independent risk factors for mortality, and it was observed that bacteremia was independent of the degree of liver failure and severity of disease. ${ }^{47}$

In a study by Jalan et al involving 497 cirrhotic patients with ACLF who developed organ failure, it was found that the group of patients admitted for decompensation in the previous 6 months had higher mortality than those without a history of decompensation (78\% versus 34\%). ${ }^{48}$ This study suggests that previous episodes of decompensation (multiple injury) and lack of recovery of liver function compared with the baseline condition (reduction of critical mass) are prognostic factors in patients with ACLF.

In the EASL-CLIF Consortium study, factors predicting mortality in patients with ACLF were elevated serum leukocytes, elevated CLIF-SOFA score, and absence of previous liver decompensation. ${ }^{11}$ An overall short-term mortality of $33.9 \%$ was observed in patients with ACLF at baseline, with a mortality of $29.7 \%$ when the patients developed ACLF during follow-up and $1.9 \%$ for cirrhotic patients without ACLF. The mortality rate was directly proportional to the number of organ failures observed (Table 2).

\section{Reversibility}

The main characteristic that distinguishes ACLF from decompensated cirrhosis is total or partial regression of liver function to the prior baseline condition, as long as the diagnosis is made in a timely manner and therapeutic maneuvers to control the precipitating factor are started immediately. Extrapolating the results observed in the work of Jalan et al, it was found that up to $50 \%$ of patients with ACLF had a reversible factor, ${ }^{48}$ and patients with ACLF have a high short-term mortality but

Table 2

\begin{tabular}{lll}
\hline Subgroups & $\begin{array}{l}\text { Mortality rate } \\
\text { at } 28 \text { days }\end{array}$ & $\begin{array}{l}\text { Mortality rate } \\
\text { at } \mathbf{9 0} \text { days }\end{array}$ \\
\hline ALD alone ${ }^{\mathrm{a}}$ & $4.7 \%$ & $14 \%$ \\
ACLF grade I & $22.1 \%$ & $40.7 \%$ \\
ACLF grade 2 & $32 \%$ & $52.3 \%$ \\
ACLF grade 3 & $76.7 \%$ & $79.1 \%$ \\
\hline
\end{tabular}

Notes: CLIF-SOFA criteria: ${ }^{\text {aALD }}$ without organ failure or ALD with only one organ failure (liver, circulatory, or respiratory failure, or coagulopathy), other than kidney failure, and without encephalopathy, or ALD and brain failure with serum creatinine $\leq 1.5 \mathrm{mg} / \mathrm{dL}$. Data from Moreau et al. ${ }^{\prime \prime}$

Abbreviations: ACLF, acute-on-chronic liver failure; ALD, acute liver disease; CLIF, Chronic Liver Failure; SOFA, Sequential Organ Failure Assessment. those who survived the acute exacerbation show a long-term outcome comparable to that of patients with decompensated cirrhosis, as observed in the study of Katoonizadeh et al. ${ }^{49}$

\section{Treatment}

Central to the treatment of ACLF is early diagnosis and control of the precipitating factor. However, one of the main limitations in the treatment of these patients is our limited knowledge about the pathophysiology of this syndrome. Moreover, due to its multifactorial nature, a single therapeutic action would not be effective in reversing the disease.

The main principles of management in these patients are to provide support to each of the organs with failure and to promote recovery of the hepatic gland. Regarding liver support systems, there are devices that attempt to provide a detoxification function in patients with ACLF until liver transplantation is done or recovery of liver function takes place. There are two main types of devices, ie, those based on acellular liver support systems (such as albumin dialysis devices and plasma exchange devices) and cell-based devices.

A variety of studies have analyzed the utility of acellular liver support systems (molecular adsorbent recirculating system, Prometheus, extracorporeal detoxification systems for supportive therapy) in patients with ACLF, although the results are still controversial and so far there is no conclusive evidence. The role of these systems as a bridge treatment to liver transplantation or while the precipitating event is reversed is still being evaluated. ${ }^{1,13,50-52}$

Recently, the impact of the molecular adsorbent recirculating system in association with standard care has been assessed in 189 patients diagnosed with ACLF compared with an ACLF group assigned to standard care only. Although the group randomized to the molecular adsorbent recirculating system showed a decrease in bilirubin and creatinine levels (the decrease was only statistically significant for bilirubin) and improvement in the degree of encephalopathy, no significant difference in transplantation-free survival was found, and survival at 28 and 90 days was similar in both groups. ${ }^{21}$ The lack of correlation between biochemical improvement, mortality, and transplantation-free survival may be explained by the duration of dialysis. Moreover, it has been reported that the molecular adsorbent recirculating system lacks the ability to remove toxic substances in a single session. ${ }^{53}$

An unpublished study found that a cellular device supporting patients with ACLF induced by viral reactivation of hepatitis B virus was useful in prolonging survival, ${ }^{54}$ but the final results of this study are needed to recommend its use in this specific group. 
Garg et a ${ }^{55}$ recently investigated the possibility of liver regeneration in a small group of patients with ACLF using bone marrow stem cells previously mobilized by granulocyte colony-stimulating factor; this treatment was associated with significantly improved survival and liver function (reduction of MELD and SOFA scores, and prevention of sepsis, hepatorenal syndrome, and hepatic encephalopathy). These results need to be replicated and confirmed in other populations.

Liver transplantation is the only curative option that can salvage ACLF patients, but data on liver transplantation for patients with this entity are scarce, and the timing and indication for transplantation are not well defined. Where the time of transplantation is a critical element in the patient's prognosis, living donor transplantation is an attractive option. Promising results in transplantation for patients with ACLF induced by reactivation of hepatitis B virus have been observed, with a study from a single institution reporting a survival rate of $80 \%$ at 5 years in such patients. ${ }^{56} \mathrm{~A}$ prospective study in a Chinese population, which analyzed preoperative, intraoperative, and postoperative survival in 100 liver transplant patients diagnosed with ACLF receiving donation from cadavers or living donors, reported a total mortality rate of $20 \%$ (survival rates of $76.8 \%, 75.6 \%$, and $74.1 \%$, at one, 3 , and 5 years, respectively). ${ }^{57}$

In a study from the People's Republic of China ${ }^{58}$ in which the King's College Hospital criteria ${ }^{59}$ was used to prioritize organ allocation, liver transplantation was most often done with living donor liver donation and the long-term survival rates were satisfactory. The authors concluded that the King's College Hospital criteria may be applicable for exacerbation of a previous hepatopathy, particularly when hepatitis B is the cause, in contrast with other causes of cirrhosis where the criteria may not be applicable. More recently, Finkenstedt et a ${ }^{60}$ retrospectively evaluated 104 patients with a diagnosis of ACLF (by APASL criteria), 65\% of whom were evaluated for liver transplant, and 23\% were finally transplanted from a deceased donor, resulting in a wait list mortality rate of $54 \%$. The mortality rates at one year and 5 years were $87 \%$ and $82 \%$, respectively, and comparable with rates for non-ACLF patients; however, liver transplant was feasible in less than one quarter of the patients (the poor prognosis of patients with ACLF was mainly attributable to infectious complications and sepsis). The majority of patients underwent transplantation during their first hospitalization after a median wait time of 24 (range 5-115) days, with sepsis and hemorrhagic shock being the main causes of death during the wait list period. Patients who successfully underwent liver transplantation had better renal function, lower MELD scores, and lower serum C-protein levels at admission. No differences between transplant and nontransplant patients were seen with respect to underlying liver disease, cause of decompensation, presence of hepatocellular carcinoma, sex, age, ascites, hepatorenal syndrome, bilirubin, serum sodium, International Normalized Ratio, or albumin. In light of these encouraging results, further prospective multicenter studies should be carried out to define the characteristics of ACLF patients who will benefit from liver transplantation.

Pretransplant infection control seems to have a positive impact in ACLF patients undergoing living donor transplantation, as demonstrated by Lin et $\mathrm{al}^{61}$ in a study involving 54 patients with ACLF undergoing liver transplantation, 34 of whom had pretransplant infection. No differences were found between the group with pretransplant infection controlled with antibiotics and the group without infection with regard to one-year post-transplant survival, graft rejection, length of stay in the intensive care unit, and post-transplant infection rates.

Antiviral therapy should be considered in patients with ACLF associated with reactivation of hepatitis B virus or acute viral infection in the setting of chronic liver disease. ${ }^{62}$ A prospective study in India found that use of tenofovir was associated with an improved survival rate in patients with ACLF after 3 months, with the tenofovir-treated group having a survival rate of $57 \%$ compared with $15 \%$ in the placebo group ( $P=0.03$ ); however, this study is limited by its small sample size ${ }^{63}$ Further prospective studies will be needed to determine the efficacy of nucleoside analog therapy in patients with ACLF.

\section{Conclusion}

ACLF is a devastating event with very high short-term mortality. Although its definition is not well established, it appears that the degree and number of failing organs are the main factors determining the outcome (mortality) of patients with ACLF, and not the degree of liver disease determined by conventional scores. Recently, the EASL-CLIF Consortium proposed new classification criteria, and only validation and comparison of the results found by this group can tell us whether the entity called ACLF necessarily corresponds to a single entity, or if it includes groups that reflect different entities, each one associated with a different prognosis, where the most sensitive organ predicting mortality is the kidney and not the liver, indicating that the pathophysiological mechanisms underlying this syndrome are likely to be different to the traditional view of "decompensation". Pathophysiology can be explained using a concept similar 
to the PIRO system used to describe the pathophysiology in sepsis.

Prospective studies are needed to identify patients with chronic liver disease at risk of developing ACLF, in whom treatment strategies are compared depending on the affected organ and the impact of these strategies on survival rate are evaluated. Finally, liver transplant groups must define the characteristics of patients with ACLF who could benefit from this therapy, although it seems that the results are very similar to those in the cirrhotic population.

\section{Disclosure}

The authors report no potential conflicts of interest relevant to this work.

\section{References}

1. Hassanein T, Schade R, Hepburn I. Acute-on-chronic liver failure: extracorporeal liver assist devices. Curr Opin Crit Care. 2011;17: 195-203.

2. D'Amico G, Garcia-Tsao G. Natural history and prognostic indicator of survival in cirrhosis: a systematic review of 118 studies. $J$ Hepatol. 2006;44:217-231

3. Garcia-Tsao G, Lim F. Management and treatment of patients with cirrhosis and portal hypertension: recommendations from the Department of Veterans Affairs Hepatitis C Resource Center Program and the National Hepatitis C Program. Am J Gastroenterol. 2009;104:1802-1829.

4. García-Tsao G, Friedman S, Iredale J, Pinzani M. Now there are many (stages) where before there was one: in search of a pathophysiological classification of cirrhosis. Hepatology. 2010;51:1445-1449.

5. Arvaniti V, D'Amico G, Fede G, et al. Infections in patients with cirrhosis increase mortality four-fold and should be used in determining prognosis. Gastroenterology. 2010;139:1246-1256.

6. Sen S, Williams R, Jalan R. The pathophysiological basis of acute-onchronic liver failure. Liver. 2002;22 Suppl 2:5-13.

7. Jalan R, Williams R. Acute-on chronic liver failure: pathophysiological basis of therapeutic options. Blood Purif. 2002;20:252-261.

8. Aithal G. Defining 'acute on chronic liver failure': an identity crisis! Indian J Gastroenterol. 2010;29:177-180.

9. Kumar A, Das K, Sharma P, Mehta V. Hemodynamic studies in acuteon-chronic liver failure. Dig Dis Sci. 2009;54:869-878.

10. Liu Q. Role of cytokines in the pathophysiology of acute-on-chronic liver failure. Blood Purif. 2009;28:331-341.

11. Moreau R, Jalan R, Gines P, et al. Acute on chronic liver failure is a distinct syndrome that develops in patients with acute decompensation of cirrhosis. Gastroenterology. 2013;144:1426-1437.

12. Ohnishi H, Sugihara J, Moriwaki H, Muto Y. Acute-on-chronic liver failure. Ryoikibetsu Shokogun Shirizu. 1995;7:217-219.

13. Sarin S, Kumar A, Almeida J, et al. Acute-on-chronic liver failure: consensus recommendations of the Asian Pacific Association for the study of the liver (APASL). Hepatol Int. 2009;3:269-282.

14. Olson J, Kamath P. Acute-on-chronic liver failure: concept, natural history, and prognosis. Curr Opin Crit Care. 2011;17:165-169.

15. Duseja A, Chawla Y, Dhiman R, Kumar A, Choudhary N, Taneja S. Non-hepatic insults are common acute precipitants in patients with acute on chronic liver failure (ACLF). Dig Dis Sci. 2010;55:3188-3192.

16. Rolando N, Wade J, Davalos M, Wendon J, Philpott-Howard J, Williams R. The systemic inflammatory response syndrome in acute liver failure. Hepatology. 2000;32:734-739.
17. Tilg H, Wilmer A, Vogel W, et al. Serum levels of cytokines in chronic liver disease. Gastroenterology. 1992;103:264-274.

18. Moreno RP, Metnitz B, Adler L, Hoechtl A, Bauer P, Metnitz PG; SAPS 3 Investigators. Sepsis mortality prediction based predisposition, infection and response. Intensive Care Med. 2008;34:496-504.

19. Jalan R, Gines P, Olson JC, et al. Acute-on-chronic liver failure. J Hepatol. 2012;57:1336-1348.

20. Plataki M, Hubmayr RD. The physical basis of ventilator-induced lung injury. Expert Rev Respir Med. 2010;4:373-385.

21. Bañares R, Nevens F, Larsen FS, et al. Extracorporeal albumin dialysis with the molecular adsorbent recirculating system in acute on chronic liver failure: the RELIEF trial. Hepatology. 2013;57:1153-1162.

22. Elsing C, Harenberg S, Stremmel W, Thomas H. Serum levels of soluble Fas, nitric oxide and cytokines in acute decompensated cirrhotic hepatic patients. World J Gastroenterol. 2007;13:421-425.

23. Guicciardi M, Gores G. Apoptosis: a mechanism of acute and chronic liver injury. Gut. 2005;54:1024-1033.

24. Lemasters J. Necroapoptosis and the mitochondrial permeability transition: shared pathways to necrosis and apoptosis. Am J Physiol. 1999;276:1-6.

25. Wasmuth H, Kunz D, Yagmur E, et al. Patients with acute on chronic liver failure display 'sepsis-like' immune paralysis. J Hepatol. 2005;42: 195-201.

26. Mookerjee R, Stadlbauer V, Lidder S, et al. Neutrophil dysfunction in alcoholic hepatitis superimposed on cirrhosis is reversible and predicts outcome. Hepatology. 2007;46:831-840.

27. Stadlbauer V, Mookerjee RP, Hodges S, et al. Effect of probiotic treatment on deranged neutrophil function and cytokine responses in patients with compensated alcoholic cirrhosis. J Hepatol. 2008;48: 945-951.

28. Garcia-Tsao G, Wiest R. Gut microflora in the pathogenesis of the complications of cirrhosis. Best Pract Res Clin Gastroenterol. 2004;18: 353-372.

29. Wiest R, Garcia-Tsao G. Bacterial translocation (BT) in cirrhosis. Hepatology. 2005;41:422-433.

30. Linderoth G, Jepsen P, Schonheyder HC, et al. Short term prognosis of community acquired bacteremia in patients with liver cirrhosis or alcoholism: a population based cohort study. Alcohol Clin Exp Res. 2006;30:636-641

31. Rastogi A, Bihari C, Maiwall R, et al. Hepatic stellate cells are involved in the pathogenesis of acute on chronic liver failure (ACLF). Virchows Arch. 2012;461:393-398.

32. Katoonizadeh A, Laleman W, Verslype C, et al. Early features of acute on chronic liver failure: a prospective cohort study. Gut. 2010;59: $1455-1456$

33. Vlachogiannakos J, Saveriadis AS, Viazis N, et al. Intestinal decontamination improves liver haemodynamics in patients with alcohol related decompensated cirrhosis. Aliment Pharmacol Ther. 2009;29: 992-999.

34. Holt S, Goodier D, Marley R, et al. Improvement in renal function in hepatorenal syndrome with N acetyl cysteine. Lancet. 1999;353: 294-295.

35. Akriviadis E, Bolta R, Briggs W, et al. Pentoxyfilline improves short term survival in severe alcoholic hepatitis: a double blind placebo controlled trial. Gastroenterology. 2000;119:1637-1648.

36. Córdoba J, García-Martinez R, Simón-Talero M. Hyponatremic and hepatic encephalopathies: similarities, differences and coexistence. Metab Brain Dis. 2010;25:73-80.

37. Fichet J, Mercier E, Genee O, et al. Prognosis and 1 year mortality of intensive care unit patients with severe liver encephalopathy. J Crit Care. 2009;24:364-370.

38. Ruiz del Arbol L, Monescillo A, Arocena C, et al. Circulatory function and hepatorenal syndrome in cirrhosis. Hepatology. 2005;42: 439-447.

39. Mookerjee R. Acute-on-chronic liver failure: the liver and portal haemodynamics. Curr Opin Crit Care. 2011;17:170-176. 
40. García-Martínez R, Córdoba J. Acute-on-chronic liver failure: the brain. Curr Opin Crit Care. 2011;17:177-183.

41. Liu H, Lee S. Acute-on-chronic liver failure: the heart and systemic hemodynamics. Curr Opin Crit Care. 2011;17:190-194.

42. Cárdenas A, Ginès P. Acute-on-chronic liver failure: the kidneys. Curr Opin Crit Care. 2011;17:184-189.

43. Laleman W, Williams R, Evenepoel P, Verslype C, Fevery J, Nevens F. Non-biological liver support in liver failure. Aliment Pharmacol Ther. 2006;23:351-363.

44. Laleman W, Wilmer Al, Evenepoel P, et al. Effect of the molecular adsorbent recirculating system and Prometheus devices on systemic haemodynamics and vasoactive agents in patients with acute-on-chronic alcoholic liver failure. Crit Care. 2006;10:R108.

45. Stadlbauer V, Davies N, Sen S, Jalan R. Artificial liver support systems in the management of complications of cirrhosis. Semin Liver Dis. 2008;28:96-109.

46. Cholangitas E, Senzolo M, Patch D, Shaw S, Hui C, Burroughs A. Review article: scoring systems for assessing prognosis in critically ill adult cirrhotics. Aliment Pharmacol Ther. 2006;24:453-454.

47. Karvellas C, Pink F, McPhail M, et al. Bacteremia, acute physiology and chronic health evaluation ii and modified end stage liver disease are independent predictors of mortality in critically ill nontransplanted patients with acute on chronic liver failure. Crit Care Med. 2010;38:121-126.

48. Jalan R, Stadlbauer V, Sen S, et al. Natural history of acute decompensation of cirrhosis: the basis of the definition, prognosis, and pathophysiology of acute-on-chronic liver failure. Hepatology. 2006; 44 Suppl 1:371A-372A.

49. Katoonizadeh A, Laleman W, Verslype C, et al. Early features of acuteon-chronic alcoholic liver failure: a prospective cohort study. Gut. 2010;59:1561-1569.

50. Kjaergard L, Liu J, Als-Nielsen B, Gluud C. Artificial and bioartificial support systems for acute and acute-on-chronic liver failure: a systematic review. JAMA. 2003;289:217-222.

51. Khuroo M, Khuroo M, Farahat K. Molecular adsorbent recirculating system for acute and acute-on-chronic liver failure: a meta-analysis. Liver Transpl. 2004;10:1099-1106.

52. Hessel F, Bramlage P, Wasem J, Mitzner S. Cost-effectiveness of the artificial liver support system MARS in patients with acute-on-chronic liver failure. Eur J Gastroenterol Hepatol. 2010;22:213-220.
53. Evenepoel P, Laleman W, Wilmer A, et al. Prometheus versus molecular adsorbents recirculating system: comparison of efficiency in two different liver detoxification devices. Artif Organs. 2006;30: 276-284.

54. Zhong-Ping Duan JZ, Xin S, Ju Chen M, et al. Interim results of randomized controlled trial of ELAD in acute on chronic liver disease. Hepatology. 2007;46:274A.

55. Garg V, Garg H, Khan A, et al. Granulocyte colony stimulating factor mobilizes CD34+ cells and improves survival of patients with acute on chronic liver failure. Gastroenterology. 2012;142:505-512.

56. Chen Z, Wen T, Zeng Y, et al. A single institution experience with living donor liver transplantation for acute on chronic hepatitis B liver failure. Hepatogastroenterology. 2011;58:1267-1273.

57. Duan BW, Lu SC, Wang ML, et al. Liver transplantation in acute on chronic liver failure patients with high model for end stage liver disease (MELD) scores: a single center experience of 100 consecutive cases. J Surg Res. 2013;183:936-943.

58. Chan AC, Fan ST, Lam BK. Liver transplantation for acute on chronic liver failure. Hepatol Int. 2009;3:571-581.

59. Neuberger J, Gimson A, Davies M et al. Selection of patients for liver transplantation and allocation of donated livers in the UK. Gut. 2008;57:252-257.

60. Finkenstedt A, Nachbaur K, Zoller H, et al. Acute on chronic liver failure: excellent outcomes after liver transplantation but high mortality on the wait list. Liver Transpl. 2013;19:879-886.

61. Lin KH, Liu JW, Chen CL, et al. Impacts of pretransplant infections on clinical outcomes of patients with acute-on-chronic liver failure who received living donor liver transplantation. PLos One. 2013;8: $1-7$.

62. Vizzutti F, Arena U, Laffi G, Marra M. Acute-on-chronic liver failure: from pathophysiology to clinical management. Trends Anaesth Crit Care. 2013;3:122-129.

63. Garg H, Sarin SK, Kumar M, Garg V, Sharma BC, Kumar A. Tenofovir improves the outcome in patients with spontaneous reactivation of hepatitis B presenting as acute on chronic liver failure. Hepatology. 2011;53:774-780.
Therapeutics and Clinical Risk Management

\section{Publish your work in this journal}

Therapeutics and Clinical Risk Management is an international, peerreviewed journal of clinical therapeutics and risk management, focusing on concise rapid reporting of clinical studies in all therapeutic areas, outcomes, safety, and programs for the effective, safe, and sustained use of medicines. This journal is indexed on PubMed Central, CAS,

\section{Dovepress}

EMBase, Scopus and the Elsevier Bibliographic databases. The manuscript management system is completely online and includes a very quick and fair peer-review system, which is all easy to use. Visit http://www.dovepress.com/testimonials.php to read real quotes from published authors. 